\title{
Balance control of the orthostatic body pose in physical education of students
}

\author{
Ihor P. Zanevskyy ${ }^{1 \mathrm{CDE}}$, Stanislaw Nowak ${ }^{2 \mathrm{ABE}}$ \\ ${ }^{1}$ Lviv State University of Physical Culture, Lviv, Ukraine \\ ${ }^{2}$ Technological and Humanistic University in Radom, Poland
}

Authors' Contribution: A - Study design; B - Data collection; C - Statistical analysis; D - Manuscript Preparation; E - Funds Collection

\begin{abstract}
Purpose: $\quad$ Maintaining upright body posture is fundamental for humans. The aim of the research is to determine influence of the feet situation on the balance of the orthostatic body pose in physical education.

Material: $\quad$ Ten 19-21 years old university students were involved into the research. The test consisted of five measurements - each of them in another feet situation with ten minutes relax before every measurement. Measurements were done using two twin platforms kinesis-meter CQStab2P. A length of the horizontal projection of centre of pressure during 30 seconds was measured as a test result.

Results: $\quad$ Because near normal distribution $(S W-W=0.893-0.963, p=0.183-0.806)$, parametric statistics were used to elaborate results on the balance test reliability: two ways ANOVA, intra-class correlation, $t$-Student, and Pearson correlation. Groups variations of results in all the five feet situations were derived about the border between moderate and great: $V=20.0-23.5 \%$. Test-retest results correlate between groups repeated measurement on moderate - high levels $(r=0.593-0.954)$.

Conclusions: In the balance control of the orthostatic body pose in the physical education of students a feet situation should be taken into consideration, because a significant difference between lengths of the centre of pressure during the control on the balance platform relatively a body pose $(p<.046)$. Balance testing on the platform could be conducted on the appropriable, good, and excellent levels of reliability using intra-class correlation coefficient $($ ICC $=0.791-0.975)$.

Keywords: postural stability, human body pose, centre of weight, test reliability, balance platform.
\end{abstract}

\section{Introduction}

Scientific researches of human body standing have a long history. Physiological foundations of the balance control have been created $[1,2]$. Taking into account difficulties of remaining of a human body in steady vertical situation on a small support area, researchers draw attention on the complicated physiological mechanism of the postural stability [3].

Oscillations of the human body in the standing pose is not a result of passive internal processes or external influences, but a result of the activity of the physiological mechanism of stabilisation and dynamic control of centre of gravity in the space. During the development of the human organism, its control function regarding stabilisation of the standing pose is one of the core problems in motor control science [4].

Maintaining upright body posture is fundamental for humans. Therefore, research concerning balance control and postural stability is very diverse. Several clinical and laboratory methods have been developed which enable researchers to asses different dimensions of the postural control system. Centre of pressure emerges as the most common variable among others registered with the use of force plates. It is defined as the point of application of ground reaction forces under the feet. Centre of pressure is the outcome of the inertial forces of the body and

(c) Ihor P. Zanevskyy, Stanislaw Nowak, 2020

doi:10.15561/20755279.2020.0108 equilibrium restoring forces of the postural control system $[5$, p. 39].

Increasing leg strength, leg power and overall balance can improve mobility and reduce fall risk. Sensor-based assessment of peak power during the sit-to-stand transfer may be useful for detecting changes in mobility and fall risk. Therefore, the study [6] investigated whether sensorbased sit-to-stand peak power and related measures are sensitive to the effects of increasing leg strength, leg power and overall balance in older adults.

Balance and resistance training can improve healthy older adults' balance and muscle strength. Delivering such exercise programs at home without supervision may facilitate participation for older adults because they do not have to leave their homes. To date, no systematic literature analysis has been conducted to determine if supervision affects the effectiveness of these programs to improve healthy older adults' balance and muscle strength / power. Lacroix et al. compared effects of supervised vs. unsupervised training programs on balance and muscle strength in older adults. They noticed the positive effects of supervised training are particularly prominent when compared with completely unsupervised training programs. It was therefore recommended to include supervised sessions (i.e., two out of three sessions / week) in balance / resistance training programs to effectively improve balance and muscle strength / power in older adults [7, p. 2341]. 
Validity and reliability of limits-of-stability testing is a core problem in the research methodology of postural stability. Based on the wide range of intra-class correlation values Pickerill and Harter observed for the NeuroCom measures, clinicians and researchers alike should establish the reliability of the limits of stability testing for their own clinics and laboratories. The low to moderate reliability outcomes observed for the Biodex $[8$, p. 600] measures were not of sufficient magnitude for us to recommend using the limits of stability measures from this system as the gold standard. The moderate Pearson interclass correlations we observed suggest that the Biodex and NeuroCom postural stability systems provided unique information. In this study of healthy participants, the concurrent and construct validity of the Biodex and NeuroCom the limits of stability tests were not definitively established.

The effects of balance training in older adults on proxies of postural control and mobility are well documented in the literature. However, evidence-based dose-response relationships in the balance training modalities (i.e., training period, training frequency, and training volume) have not yet been established in healthy older adults. Lesinski et al. showed that balance training is an effective means to improve proxies of static/dynamic steady-state, proactive, and reactive balance as well as performance in balance test batteries in healthy older adults. Furthermore, they were able to establish effective balance training modalities to improve balance performance in healthy older adults. Thus, practitioners and therapists are advised to consult the identified dose-response relationships of this systematic literature review and meta-analysis. However, further research of high methodological quality is needed to determine dose-response relationships of balance training in terms of detailed information on training volume (e.g., number of exercises per training session) and a feasible and effective method to regulate training intensity in the balance training [9, p. 1721].

Effects and dose-response relationships of balance training on measures of balance are well-documented for healthy young and old adults. However, this has not been systematically studied in youth. Balance training is a highly effective means to improve balance performance with moderate to large effects on static and dynamic balance in healthy youth irrespective of age, sex, and training status, setting and testing method. Gebel et al. discussed the effects and dose-response relationship of balance training on balance performance in youth. The examined training modalities did not have a moderating effect on balance performance in healthy adolescents. Thus, they concluded that an additional but so far unidentified training modality may have a major effect on balance performance that was not assessed in the analysis. Training intensity could be a promising candidate. However, future studies are needed to find appropriate methods to assess balance training intensity [10, p. 2067].

Kiss et al. during a systematic review and metaanalysis showed exclusively small-sized correlations between types of balance performance across the lifespan.
This indicates that balance performance seems to be task-specific rather than a "general ability". The results suggest that for assessment / training purposes a test battery / multiple exercises should be used that include static / dynamic steady-state, proactive, and reactive types of balance. Concerning the observed significant age differences, further research is needed to investigate whether they are truly existent or if they are caused by methodological inconsistencies [11, p. 1366].

Compared with control condition, unstable surfaces is effective in improving muscle strength, power and balance in adolescents, young adults and old adults. However, inconsistent results were particularly found in adolescents and young adults when the specific effects of unstable surfaces were compared with those of stable surfaces. Behm et al. reviewed the effects of strength training using unstable surfaces on strength, power and balance performance across the lifespan [12, p. 1645]. They concluded that the performance of unstable surfaces compared with stable surfaces has limited extra effects on muscle strength, power and balance performance in healthy adolescents and young adults. Given that the systematic search did not identify studies that examined the effects of unstable surfaces versus stable surfaces in children, middle-aged adults and old adults, further research of high methodological quality is needed to determine whether there are additive effects of unstable surfaces as compared with stable surfaces in those age groups.

The risk of sustaining a fall is particularly high in children and seniors. Deficits in postural control and muscle strength either due to maturation, secular declines or biologic aging are two important intrinsic risk factors for falls. During life span, performance in variables of static postural control follows a U-shaped curve with children and seniors showing larger postural sway than healthy adults. Granacher et al. created an intergenerational approach in the promotion of balance and strength for fall prevention. Measures of dynamic postural control (i.e. gait speed) as well as isometric (i.e. maximal strength) and dynamic muscle strength (i.e. muscular power) follow an inverted U-shaped curve during life span, again with children and seniors showing deficits compared to adults [13, p. 304].

Balance, strength and power relationships may contain important information at various maturational stages to determine training priorities. Hammami et al. studied associations between balance and muscle strength, power performance in male youth athletes of different maturity status. The associations between balance and muscle strength/power measures in youth athletes that increase with maturity may imply transfer effects from balance to strength/power training and vice versa in youth athletes [14, p. 521].

Traditionally, balance and/or lower extremity resistance training were used to mitigate these age-related deficits. However, the effects of resistance training are limited and poorly translate into improvements in balance, functional tasks, activities of daily living, and fall rates. 
Granacher et al. discussed the importance of trunk muscle strength for balance, functional performance, and fall prevention in seniors. A systematic approach was used to evaluate the 582 articles identified for initial review. They declared core strength training and / or Pilates exercise training as an adjunct or even alternative to traditional balance and / or resistance training programs for old adults. Further, core strength training and Pilates exercise training are easy to administer in a group setting or in individual fall preventive or rehabilitative intervention programs because little equipment and space is needed to perform such exercises. [15, p. 627]

Taking into account significance of the balance mechanism in the human ontogenesis, a problem of checking of displacement of the centre of the human body is very important in physical education of students.

Research hypothesis: a feet situation should be taken into consideration in the balance control of the orthostatic body pose in physical education.

Purpose: the aim of the research was to determine influence of the feet situation on the balance of the orthostatic body pose in physical education.

\section{Material and Methods}

\section{Participants}

Ten 19-21 years old university students were involved into the research (body mass: $78.6 \pm 14.4 \mathrm{~kg}$, body length: $180.9 \pm 8.4 \mathrm{~cm})$. All the participants were good healthy with no vestibular or visual disabilities or lower limb impairments. They trained according the program of physical education students.

This study was approved in advance by Ethical Committee of Technological and Humanistic University in Radom. The participant voluntarily provided written informed consent before participating. The procedures followed were in accordance with the ethical standards of the Ethical Committee on human experimentation.

\section{Research Design}

Measurements were done before noon in the kinesiology laboratory after a common warm up. The test consisted of five measurements - each of them in another feet situation with ten minutes relax before every measurement; five trials was completed by each participant in each of the feet situations. Measurements were done using two twin platforms kinesis-meter CQStab2P (Figure 1). A patient was asked to remain a vertical standing pose on the balance platform. A length of the horizontal projection of centre of pressure during 30 seconds was measured as a test result [16].

Two twin platforms kinesis-meter CQStab2P determined 5 feet situations (see Figure 1b): A-B, A-C, A-D, C-B, and D-B. For example, the feet situation $\mathrm{A}-\mathrm{C}$ is presented on the photo (see Figure 1a). Situation of the left foot was marked with the first letter and situation of the right - with the second.

Statistical analysis

Shapiro - Wilk test was used to evaluate probability of a normal distribution of results showed by the group on the balance platform [17]. The results were elaborated using parametric statistics of centre and variation.

Variation of measurements for each of five feet situations was estimated with the coefficient of variation:

$$
V=\frac{S D}{M} 100 \% \text {, }
$$

where $S D$ : standard deviation, $M$ : arithmetic mean. When $\mathrm{V}<10 \%$, variation is small, $10-20 \%$ - moderate, and V $>20 \%$ - great [18].

Reliability of testing was studied in the frames of intraclass correlation [19]:

$$
I C C=\frac{M S_{W}-M S_{B}}{M S_{W}},
$$

where $M S_{W}$ and $M S_{B}$ are within and between groups variations. The first one is the variance between subjects and the second - between measurements with interaction:

$$
M S_{B}=\frac{S S_{M}+S S_{I}}{(k-1)+(n-1)(k-1)},
$$

where $S S_{M}$ and $S S_{I}$ are sums of squares between measurements and interaction variations, $k$ is number of measurements, and $n$ - number of subjects.

Confidence interval limits (with $q=1-p$ ) for the reliability coefficient estimated using the intraclass correlation model (Eq. 4) were estimated as follow [19]:

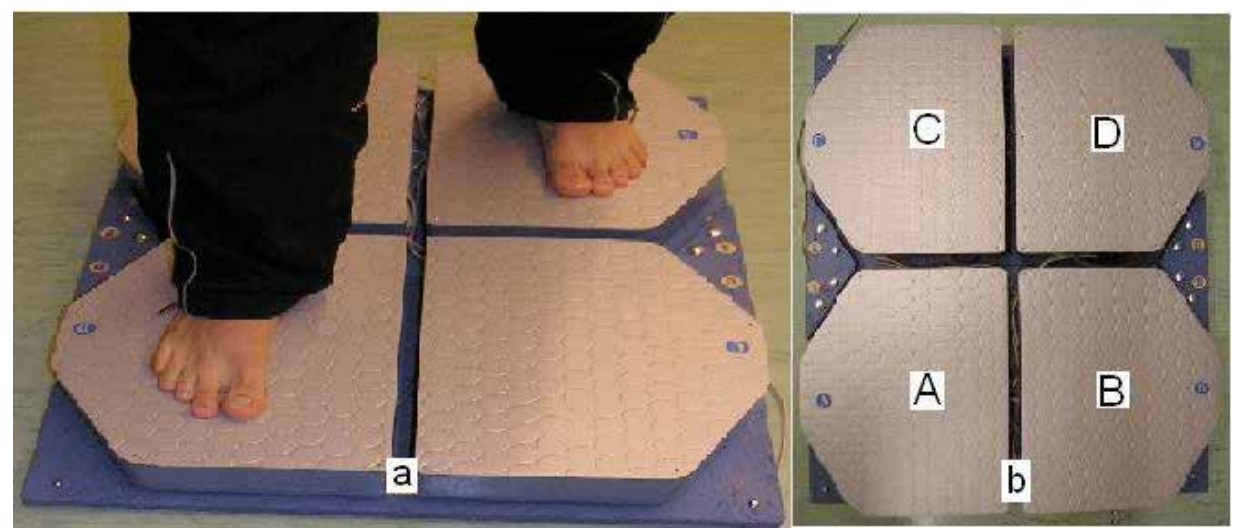

Figure 1. Two twin platforms kinesis-meter CQStab2P used for measurements [16]: testing in A-D feet situation (a), plates - sight from above (b). 
$1-1 / F_{L}$ and $1-1 / F_{U}$,

where $F_{L}=F_{0} / F_{p, n(k-1), n-1}$ and $F_{U}=F_{0} F_{p, n-1, n(k-1)}, F_{0}=M S_{W}$ $M S_{B}$, and $p$ is significance.

Test-retest reliability is a measure of the consistency of a physiological test or assessment. This kind of reliability is used to determine the consistency of a test across time. Test-retest reliability is best used for things that are stable over time, such as human motions [20]. Reliability was evaluated in the frames of reliability levels as follow: ICC $>0.95$ - excellent, $0.90-0.95$ - good, $0.80-0.90$ - moderate, $0.70-0.80$ - appropriable, $0.60-0.70-$ questionable, and $I C C<0.60$ - non appropriable.

Two ways ANOVA for repeated measurements like test-retest was used to evaluate differences between test results from different feet situations. Fisher - Snedecor F-test was used to determine significance of differences in variations between repeated measures and between patients.

Relative difference between test results measured in pairs of feet situations was calculated using the formula followed (for example, $\mathrm{A}-\mathrm{B}$ and $\mathrm{A}-\mathrm{C}$ situations):

$$
\delta l=\frac{\left|M_{A-B}-M_{A-C}\right|}{M_{A-B}+M_{A-C}} 200 \%
$$

where are arithmetic means of groups' results in corresponding pair of feet situations. Significance of the difference was determined using t-test for two paired samples.
Pearson paired correlation was used with a purpose to study test-retest interconnection between repeated measurements. The correlation power was evaluated using a common gradation of the correlation coefficient values as weak $(|r|<0.4)$, moderate $(|r|<0.4-0.7)$, and strong correlation $(|r|>0.7)$. The significance of the correlation was evaluated using t-Student parameter presented with the formula as follows:

$$
t=|r| \sqrt{\frac{n-2}{1-r^{2}}},
$$

where $r$ is coefficient of correlation, $n-$ number of participants in a group.

The computer package Statistica was used in data processing.

\section{Results}

Because near normal distribution $(S W-W=$ $0.893-0.963, p=0.183-0.806)$, parametric statistics were used to elaborate results on the balance test reliability (Table 1).

Because no significant trends $(p(F)=0.087-0.866)$ and because clear significant correlation between testretest measurements $(p(t)<0.038)$ were noticed (Table $2)$, intraclass correlation coefficient was used to evaluate reliability of this balance test (Figure 2). Corresponding results of two ways ANOVA for repeated measures regarding the A-B feet situation are collected in Table 3.

Table 1. Results on the balance test

\begin{tabular}{llllll}
\hline \multirow{2}{*}{ Parameters } & Feet situation & & & & D-B \\
\cline { 2 - 5 } & A-B & A-C & A-D & C-B & 487.9 \\
\hline SD $(\mathrm{mm})$ & 265.8 & 540.1 & 444.8 & 395.9 & 97.5 \\
Max $(\mathrm{mm})$ & 62.5 & 111.6 & 104.6 & 81.3 & 761 \\
Min $(\mathrm{mm})$ & 410 & 873 & 639 & 597 & 313 \\
$V(\%)$ & 153 & 360 & 250 & 228 & 20.0 \\
SW-W & 23.5 & 20.7 & 23.5 & 20.5 & 0.893 \\
$\mathrm{p}(\mathrm{SW}-\mathrm{W})$ & 0.963 & 0.915 & 0.931 & 0.952 & 0.183 \\
$\mathrm{~F}$ & 0.806 & 0.315 & 0.460 & 0.695 & 0.379 \\
$\mathrm{p}(\mathrm{F})$ & 1.528 & 2.214 & 0.315 & 1.174 & 0.822 \\
$\mathrm{r}$ & 0.215 & 0.087 & 0.866 & 0.339 & $0.785-0.922$ \\
$\mathrm{p}(\mathrm{t})$ & $0.660-0.954$ & $0.684-0.915$ & $0.834-0.945$ & $0.593-0.934$ & 0.006 \\
$\mathrm{ICC}$ & 0.038 & 0.029 & 0.003 & 0.041 & 0.791 \\
$\mathrm{~F}_{0}$ & 0.946 & 0.954 & 0.975 & 0.938 & $4.78^{*}$ \\
$\mathrm{~F}_{\mathrm{U}}$ & $18.51^{*}$ & $21.68 *$ & $39.58^{*}$ & $16.02 *$ & 10.14 \\
$\mathrm{~F}_{\mathrm{L}}$ & 39.31 & 46.04 & 84.06 & 34.03 & 1.69 \\
ICC & 6.55 & 7.67 & 14.00 & 5.67 & 0.901 \\
$\mathrm{ICC}$ & 0.975 & 0.978 & 0.988 & 0.971 & 0.408 \\
Reliability level & good & excellent & excellent & good & appropriable \\
\hline
\end{tabular}

Note: $M$ - arithmetic mean; SD - standard deviation; Max - maximal; Min - minimal; F - Fisher - Snedecor statistics; $r$ - Pearson paired correlation coefficient; SW-W - Shapiro-Wilk parameter; ICC - intraclass correlation reliability coefficient; $I C C_{U}$ and $I C C_{L}$ - upper and lower limits of the coefficient; ${ }^{*} p<0.001$ - significance. 
Test-retest results correlate between groups repeated measurement on moderate - high levels $(r=0.593-$ 0.954). The strongest correlation was noticed in the results measured in $\mathrm{A}-\mathrm{B}$ feet situation, and the weakest - in C-B situation.

The results in the frames of the intra-class correlation model using Eqs (2-4), were calculated as follow: $I C C=$ $0.946, F_{0}=18.51$, and $p\left(F_{0}\right)<0.001$. Interval limits (with the $q=95 \%$ level of confidence) were calculated as 0.847 and $0.975\left(F_{L}=6.55, F_{0.05,40,9}=2.826, F_{U}=39.31, F_{0.05,9 \text {, }}\right.$ $\left.{ }_{40}=2.124\right)$. Results of intra-class correlation reliability regarding all the five feet situation balance show sufficient reliability for all the five feet situations (see Table 1). The best reliability was noticed in the $\mathrm{A}-\mathrm{C}$ and $\mathrm{A}-\mathrm{D}$ (as excellence), and the worst - in the $\mathrm{D}-\mathrm{B}$ feet situations (as appropriable).

Essential differences between results measured for feet situations were received (see Table 1). Maximal range of measured results was noticed in A-C feet situation $(360-873 \mathrm{~mm})$, minimal - in A-B feet situation $(153-410 \mathrm{~mm})$. The same picture was with mean values and variations: $540.1 \pm 111.6 \mathrm{~mm}$ and $265.8 \pm 62.5 \mathrm{~mm}$. Groups variations of results in all the five feet situations were derived about the border between moderate and great: $V=20.0-23.5 \%$ (Eq. 1). The greatest relative difference (Eq. 5) was noticed between results in the $\mathrm{A}-\mathrm{B}$ and $\mathrm{A}-\mathrm{C}$ feet situations $(68.1 \%, p<0.001)$ and the smallest - in the A-D and D-B feet situations: $9.3 \%, p=$ 0.046 (Table 4).

\section{Discussion}

The aim of the research was to determine influence of the feet situation on the balance of the orthostatic body pose in the physical education. The length of the horizontal projection of centre of pressure for straight standing on the balance platform was measured for different feet situations. Despite of many publications on the problem of balancing of the human body standing position, this problem was not investigated in a full measure. Petro et al. undertook a systematic literature review to identify and categorize existing objective methods of standing dynamic balancing ability assessment with an emphasis on the balancing devices and tasks [21]. Three major scientific literature databases (Science Direct, Web of Science, PLoS ONE) and additional sources were used. A variety of dynamic balancing assessment devices were identified and categorized as Solid ground, Balance board, Rotating platform, Horizontal translational platform, Treadmill, and Computerized Dynamic Posturography, but there were no balancing devices and tasks which were directed to consideration of feet situation.

Kawaishi and Domen studied relationship between dynamic balancing ability and posture-related modulation of the soleus H-reflex taking into consideration feet situation [22, p. 120]. The soleus maximal H-reflex, motor response, and background EMG activity were obtained during three postural conditions: prone, open-legged standing, and closed-legged standing. Dynamic balancing ability was assessed by testing stability while standing on a wobble board. A significant negative correlation was

Table 2. Pearson correlation coefficient (left - lower) and corresponding t-Student statistics (right - upper) derived in A-B feet situation

\begin{tabular}{llllll}
\hline Repeated measurements & $\mathbf{1}$ & $\mathbf{2}$ & $\mathbf{3}$ & $\mathbf{4}$ & $\mathbf{5}$ \\
\hline 1 & & $8,967^{* * *}$ & $4,855^{* *}$ & $4,175^{* *}$ & $2,744^{*}$ \\
2 & 0,954 & & $3,748^{* *}$ & $3,736^{* *}$ & $2,488^{*}$ \\
3 & 0,864 & 0,798 & & $7,172^{* * *}$ & $2,956^{*}$ \\
4 & 0,828 & 0,797 & 0,930 & & $3,377^{* *}$ \\
5 & 0,696 & 0,660 & 0,723 & 0,767 & \\
\hline
\end{tabular}

Note: ${ }^{*} p<0.05, t_{0.05, n-2}=2.306 ;{ }^{*} p<0.01, t_{0.01, n-2}=3.355$;

$* * * p<0.001, t_{0.001, n-2}=5.041$.

Table 3. Results of two ways ANOVA for repeated measures regarding the A-B feet situation

\begin{tabular}{lllllll}
\hline $\begin{array}{l}\text { Source of } \\
\text { dispersion }\end{array}$ & $S S$ & $d f$ & $M S$ & $F$ & $p$ & $Q \%$ \\
\hline Patients & 154337 & 9 & 17149 & $19.48^{*}$ & 0.000 & 80.6 \\
Measurements & 5380 & 4 & 1345 & $1.528^{*}$ & 0.215 & 2.8 \\
Interaction & 31686 & 36 & 880 & & & 16.6 \\
Errors & 37066 & 40 & 927 & & & 19.4 \\
Total & 191403 & 49 & 3906 & & 100.0 \\
\hline
\end{tabular}

Note: $S S$ - sum of squares, $d f$-degree of freedom, $M S$-variance, $F$ - Fisher parameter, $p$-significance, $Q$ - relative part of variation in a total, $* F_{0.05,9,36}=2.153, F_{0.05,4,36}=2.634$. 


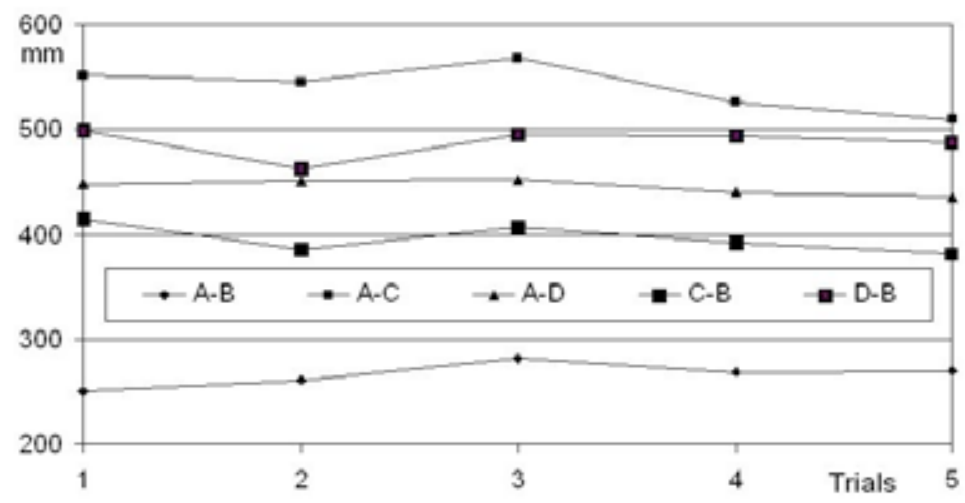

Figure 2. Balance test-retest results vs. repeated trials.

Table 4. Relative difference (left - lower, \%) and significance (right - upper)

\begin{tabular}{llllll}
\hline Feet situation & A-B & A-C & A-D & C-B & D-B \\
\hline A-B & & 0.000 & 0.000 & 0.000 & 0.000 \\
A-C & $68.1 \%$ & & 0.005 & 0.000 & 0.044 \\
A-D & $50.4 \%$ & $19.4 \%$ & & 0.102 & 0.046 \\
C-B & $39.3 \%$ & $30.8 \%$ & $11.6 \%$ & & 0.003 \\
D-B & $58.9 \%$ & $10.1 \%$ & $9.3 \%$ & $20.8 \%$ & \\
\hline
\end{tabular}

observed between balancing scores and reflex modulation from open-legged standing to closed-legged standing. This suggests that the ability to modulate monosynaptic stretch reflex excitability in response to a changing posture is a significant factor for dynamic balancing. These results are similar with results of our measurements (see Table 1), because $\mathrm{A}-\mathrm{B}$ feet situation accompanies of open-legged standing and $\mathrm{A}-\mathrm{C}, \mathrm{D}-\mathrm{B}$ feet situations accompany of closed-legged standing (see Figure 1b). Because A-D and $\mathrm{C}-\mathrm{B}$ feet situations occupy intermediate space, future studies on the subject are needed.

A reliable measure of dynamic postural control is needed for inclusion in the sports-related concussion assessment battery. Currently, there is not a clinical gold standard. The limits of stability test have potential to be a useful tool to collect objective data on important dynamic postural stability variables. Psychometric properties of the limits of stability test with healthy young adults are yet to be established. Limits of stability provide a reliable measure of dynamic postural control for young adults. Lininger et al. determined test-retest reliability of the limits of stability test performed by young adults using. They recommended two trials at baseline with the first being an adaptation trial to ensure accuracy of findings; care needs to be taken when interpreting endpoint excursion and directional control scores on post-injury tests due to a learning effect for those variables. We got very close intra-class correlation coefficients values comparing results of this research (see Table 1). Relative reliability was measured within each session with an intraclass correlation coefficient (ICC $[3, k]$ ) on each of the five dependent variables (movement velocity, directional control, maximum excursion, endpoint excursion, and reaction time) provided by the Neurocom. Test-retest reliability was assessed using a repeated-measures analysis of variance along with an $\operatorname{ICC}(3, k)$ for relative reliability. An ICC value of 0.90 or higher was defined as having a high reliability, moderate reliability for ICC values between $0.80-0.89$, and below 0.80 as questionable $[20$, p. 800$]$ that is supported with our results on balance test reliability (see Table 1).

Limitations of our study is caused by training effect of the testing balance on the platform, because trends in the repeated trials caused by combination of fatigue and training effects. Age-related postural misalignment, balance deficits and strength / power losses are associated with impaired functional mobility and an increased risk of falling in seniors. Core instability strength training involves exercises that are challenging for both trunk muscles and postural control and may thus have the potential to induce benefits in trunk muscle strength, spinal mobility and balance performance. Core instability strength training proved to be a feasible exercise program for seniors with a high adherence rate. Age-related deficits in measures of trunk muscle strength, spinal mobility, dynamic balance and functional mobility can be mitigated by Core instability strength training. This training regimen could be used as an adjunct or even alternative to traditional balance and/or resistance training [23, p. 105].

A method of elimination of systematic changes in repeated trials based on linear regression was proposed in the research. A linear function can be used as a model of learning effect in the test-retest design because accuracy of approximation may be over $97 \%$ and rather strong significance level for the slope coefficient $(p<0.002)$. The test scores could be purged of the learning effect 
using a slope coefficient of linear function approximate of means scores monotone increase in repeated trials. ICC $(1,1)$ used to the purged scores is an estimate of single trial test-score reliability [24].

The test showed good sensitivity as possibilities for differentiation of balance ability between participants, because $80.6 \%$ of dispersion was determined by variation between participants ( $p<0.001$, see Table 3$)$. Therefore the test could be used for comparative evaluation of students regarding balancing in the process of physical education.

\section{Conclusions}

In the balance control of the orthostatic body pose in the physical education of students a feet situation should be taken into consideration, because a significant difference between lengths of the centre of pressure during the control on the balance platform relatively a body pose $(p=0.046)$. Balance testing on the platform could be conducted on the appropriable, good, and excellent levels of reliability using intra-class correlation coefficient (ICC $=0.791-0.975)$.

\section{Highlights}

The feet situation has a significant influence on maintaining upright body posture that is fundamental for humans. There are five basic feet situations regarding the orthostatic body pose. The function of balance controls of the orthostatic body pose in physical education is significantly depended of the feet situation $(p<0.05)$.

Groups variations of results in all the five feet situations were derived about the border between moderate and great $(V=20.0-23.5 \%)$.

Two twin platforms kinesis-meter CQStab2P used for measurements [16] showed good validity and reliability as an apparatus of scientific provision of research in the problem of maintaining upright body posture.

\section{Acknowledgements}

Authors are grateful to the students which participated in measurements for their patience and fruitful comments regarding the individual aspects in balancing and control of the orthostatic body pose.

\section{Information on financial support}

The authors declare no financial support regarding this paper.

\section{Conflict of Interest}

The authors declare that there is no conflict of interest regarding this research.

\section{References}

1. Weber $\mathrm{W}$, Weber E. The mechanics of human motion. Gottingen: Dieterischen Buchhandlung; 1836.

2. Braune W, Fisher O. Der Gang des Menschen [The course of humans]. Leipzig: Sachsischen Gesellschaft der Wissenschaften; 1895 (In German).

3. Bernstein NA. The co-ordination and regulation of movements. Oxford: Pergamon Press; 1967.

4. Gurfinkel VS, Kots YM, Shik ML. Regulation of the human pose. Moscow: Nauka; 1965. (in Russian)

5. JurasG,SlomkaK,FredykA,SobotaG,BacikB.Evaluationofthe limits of stability balance test. JHum Kinet. 2008; 19(1):39-52. https://doi.org/10.2478/v10078-008-0003-0

6. Regterschot GR, Folkersma M, Zhang W, Baldus H, Stevens $\mathrm{M}$, Zijlstra W.Sensitivity of sensor-based sit-to-stand peak power to the effects of training leg strength, leg power and balance in older adults. Gait Posture. 2014; 39 (1): 303-307. https://doi.org/10.1016/j.gaitpost.2013.07.122

7. Lacroix A, Hortobagyi T, Beurskens R, Granacher U. Effects of supervised vs. unsupervised training programs on balance and muscle strength in older adults: a systematic review and meta-analysis. Sports Med. 2017; 47 (11): 2341-2361. https://doi.org/10.1007/s40279-017-0747-6

8. Pickerill ML, Harter RA. Validity and reliability of limitsof-stability testing: a comparison of 2 postural stability evaluation devices. J Athl Train. 2011; 46 (6): 600-606. 606. https://doi.org/10.4085/1062-6050-46.6.600

9. Lesinski M, Hortobagyi T, Muehlbauer T, Gollhofer A, Granacher U.Effects of balance training on balance performance in healthy older adults: a systematic review and meta-analysis. Sports Med. 2015; 45 (12): 1721-1738. https://doi.org/10.1007/s40279-015-0375-y

10.Gebel A, Lesinski M, Behm DG, Granacher U. Effects and dose-response relationship of balance training on balance performance in youth: a systematic review and

meta-analysis. Sports Med. 2018; 48 (9): 2067-2089. https://doi.org/10.1007/s40279-018-0926-0

11.Kiss R, Schedler S, Muehlbauer T. Associations between types of balance performance in healthy individuals across the lifespan: a systematic review and meta-analysis. Front Physiol. 2018; 9: 1366. https://doi.org/10.3389/fphys.2018.01366

12.Behm DG, Muehlbauer T, Kibele A, Granacher U. Effects of strength training using unstable surfaces on strength, power and balance performance across the lifespan: a systematic review and meta-analysis. Sports Med. 2015; 45 (12): 1645-1669. https://doi.org/10.1007/s40279-015-0384-x

13. Granacher U, Muehlbauer T, Gollhofer A, Kressig RW, Zahner L. An intergenerational approach in the promotion of balance and strength for fall prevention - a mini-review. Gerontology. 2011; 57 (4): 304-315. https://doi.org/10.1159/000320250

14.Hammami R, Chaouachi A, Makhlouf I, Granacher U, Behm DG. Associations between balance and muscle strength, power performance in male youth athletes of different maturity status. Pediatr Exerc Sci. 2016; 28 (4): 521-534. https: //doi.org/10.1123/pes.2015-0231

15.Granacher U, Gollhofer A, Hortobagyi T, Kressig RW, Muehlbauer T. The importance of trunk muscle strength for balance, functional performance, and fall prevention in seniors: a systematic review. Sports Med. 2013; 43 (7): 627-641. https://doi.org/10.1007/s40279-013-0041-1

16.Poland. Specialized Electronic Systems for Medicine Picture Diagnostics. Platform kinesis-meter CQStab2P [document on the Internet]. 2014 [cited 2019 Dec 21]. Available from: http://www.cq.com.pl

17.S hapiro SS, WilkMB.An analysis of variance test for normality (complete samples). Biometrika. 1965; 52 (3-4): 591-611. https: //doi.org/10.1093/biomet/52.3-4.591

18.Vincent WJ. Statistics in Kinesiology. Champaign, IL: 
Human Kinetics; 1995.

19. Shrout PE, Fleiss JL. Intraclass correlation: uses in assessing rater reliability. Psychol Bul. 1979; 86: 420-428.

20.Lininger MR, Leahy TE, Haug EC, Bowman TG. Test-retest reliability of the limits of stability test performed by young adults using NeuroCom ${ }^{\circledR}$ VSR Sport. Int $J$ Sports Phys Ther. 2018; 13 (5): 800-807.

21.Petro B, Papachatzopoulou A, Kiss RM. Devices and tasks involved in the objective assessment of standing dynamic balancing: A systematic literature review. PLoS One, 2017; 12 (9), e0185188. https://doi.org/10.1371/journal.pone.0185188
22.Kawaishi Y, Domen K. The relationship between dynamic balancing ability and posture-related modulation of the soleus H-reflex. J Electromyogr Kinesiol. 2016; 26: 120-124. https://doi.org/10.1016/j.jelekin.2015.11.010

23. Granacher U, Lacroix A, Muehlbauer T, Roettger K, Gollhofer A. Effects of core instability strength training on trunk muscle strength, spinal mobility, dynamic balance and functional mobility in older adults. Gerontology. 2013; 59 (2): 105-113. https://doi.org/10.1159/000343152

24.Zanevskyy I, Zanevska L. Intraclass correlation test-score reliability of a single trial.J Test Eval.2017; 45 (3): 1073-1080. https://doi.org/10.1520/JTE20150485

\section{Information about the authors:}

Zanevskyy Ihor P. (Corresponding Author); http://orcid.org/0000-0002-9326-1167; izanevsky@ukr.net; Lviv State University of Physical Culture; Department of Informatics and Kinesiology; Kostyushka 11, 79007 Lviv, Ukraine.

Nowak Stanislaw; http://orcid.org/0000-0001-7099-8804; stanislawn@gmail.com; Technological and Humanistic University in Radom; Department of Physical Culture; Chrobrego 27, Radom 26-600, Poland.

Cite this article as:

Ihor Zanevskyy, Stanislaw Nowak. Balance control of the orthostatic body pose in physical education of students. Physical education of students, 2020;24(1):63-70. https://doi.org/10.15561/20755279.2020.0108

This is an Open Access article distributed under the terms of the Creative Commons Attribution License, which permits unrestricted use, distribution, and reproduction in any medium, provided the original work is properly cited http://creativecommons.org/licenses/by/4.0/deed.en

Received: 02.01.2020

Accepted: 31.01.2020; Published: 04.02.2020 